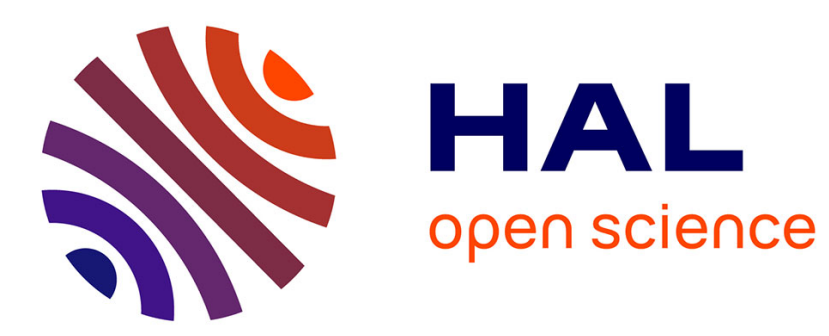

\title{
Propagation of toxic substances in the urban atmosphere: A complex network perspective
}

Sofia Fellini, Pietro Salizzoni, Lionel Soulhac, Luca Ridolfi

\section{To cite this version:}

Sofia Fellini, Pietro Salizzoni, Lionel Soulhac, Luca Ridolfi. Propagation of toxic substances in the urban atmosphere: A complex network perspective. Atmospheric Environment, 2019, 198, pp.291 301. 10.1016/j.atmosenv.2018.10.062 . hal-01925872

\section{HAL Id: hal-01925872 \\ https://hal.science/hal-01925872}

Submitted on 18 Nov 2018

HAL is a multi-disciplinary open access archive for the deposit and dissemination of scientific research documents, whether they are published or not. The documents may come from teaching and research institutions in France or abroad, or from public or private research centers.
L'archive ouverte pluridisciplinaire HAL, est destinée au dépôt et à la diffusion de documents scientifiques de niveau recherche, publiés ou non, émanant des établissements d'enseignement et de recherche français ou étrangers, des laboratoires publics ou privés. 


\section{Accepted Manuscript}

Propagation of toxic substances in the urban atmosphere: A complex network perspective

Sofia Fellini, Pietro Salizzoni, Lionel Soulhac, Luca Ridolfi

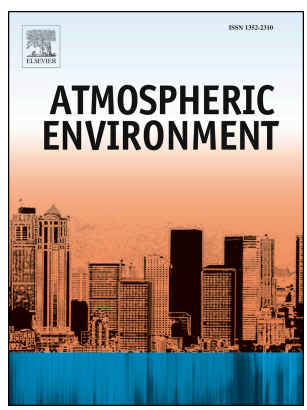

PII:

S1352-2310(18)30760-X

DOI:

https://doi.org/10.1016/j.atmosenv.2018.10.062

Reference: $\quad$ AEA 16359

To appear in: Atmospheric Environment

Received Date: 16 July 2018

Revised Date: 11 October 2018

Accepted Date: 29 October 2018

Please cite this article as: Fellini, S., Salizzoni, P., Soulhac, L., Ridolfi, L., Propagation of toxic substances in the urban atmosphere: A complex network perspective, Atmospheric Environment (2018), doi: https://doi.org/10.1016/j.atmosenv.2018.10.062.

This is a PDF file of an unedited manuscript that has been accepted for publication. As a service to our customers we are providing this early version of the manuscript. The manuscript will undergo copyediting, typesetting, and review of the resulting proof before it is published in its final form. Please note that during the production process errors may be discovered which could affect the content, and all legal disclaimers that apply to the journal pertain. 
Dispersion in the urban atmosphere
Spreading process on a complex network
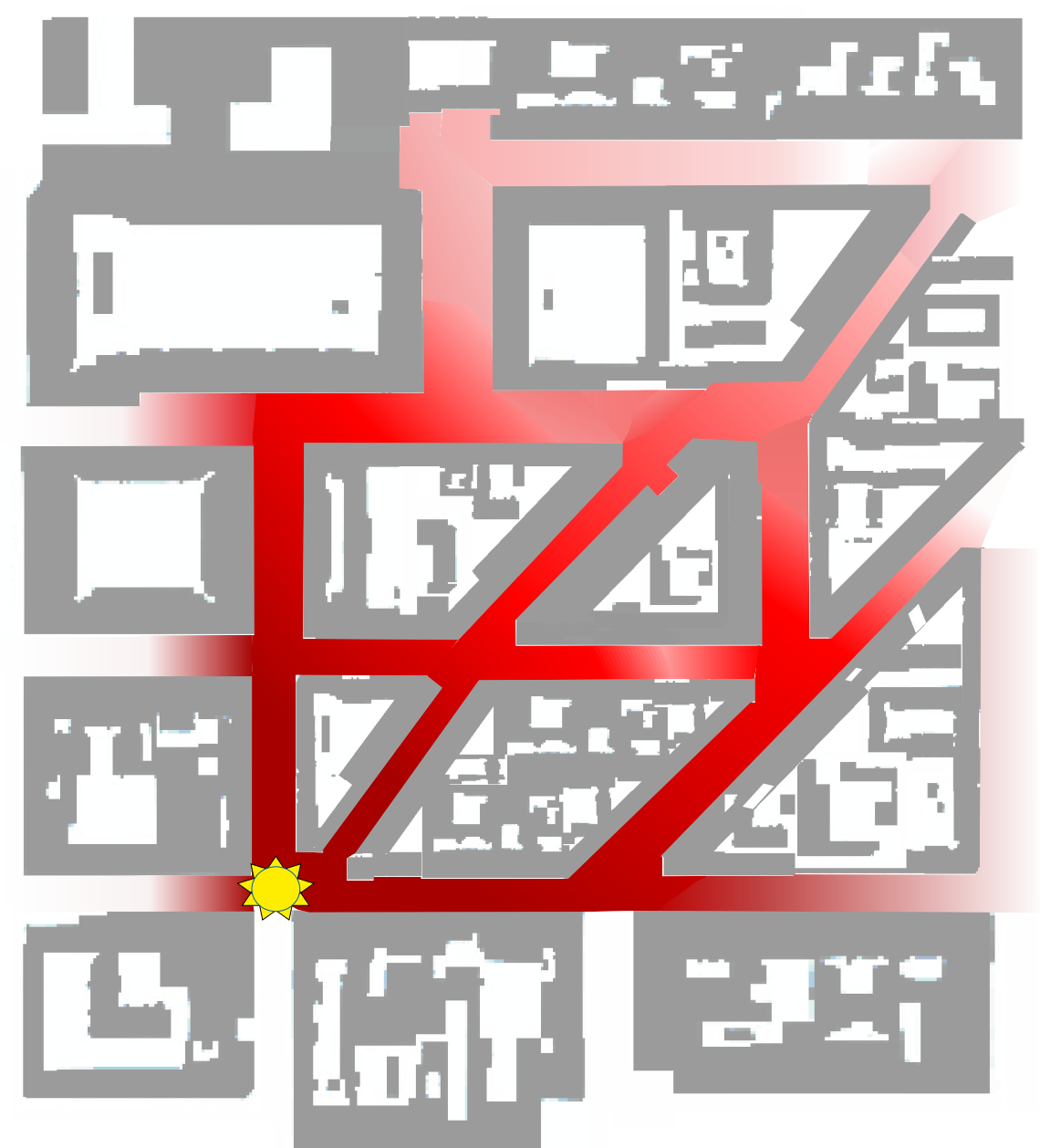

COMPLEXNETWORK

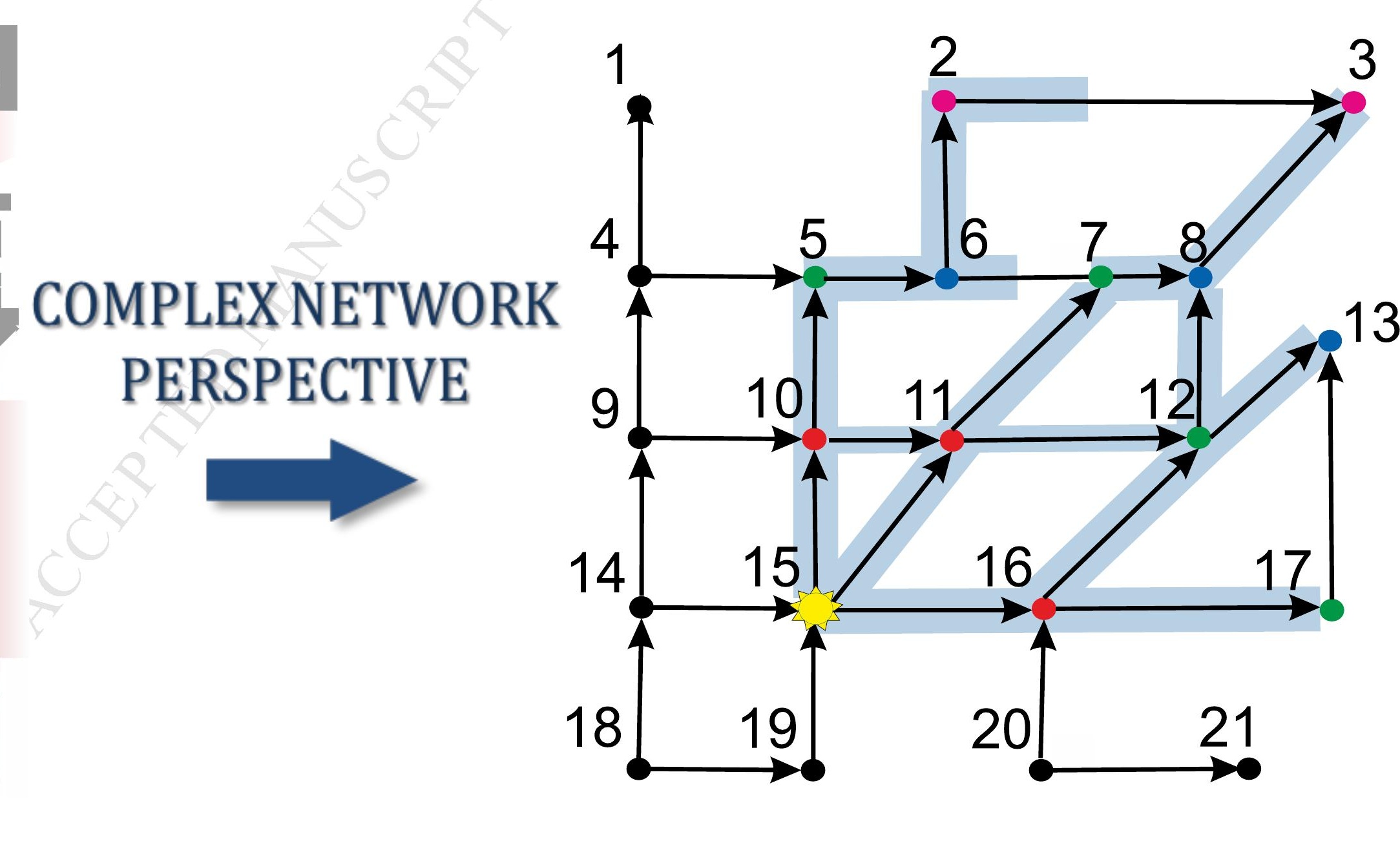
PERSPECTIVE

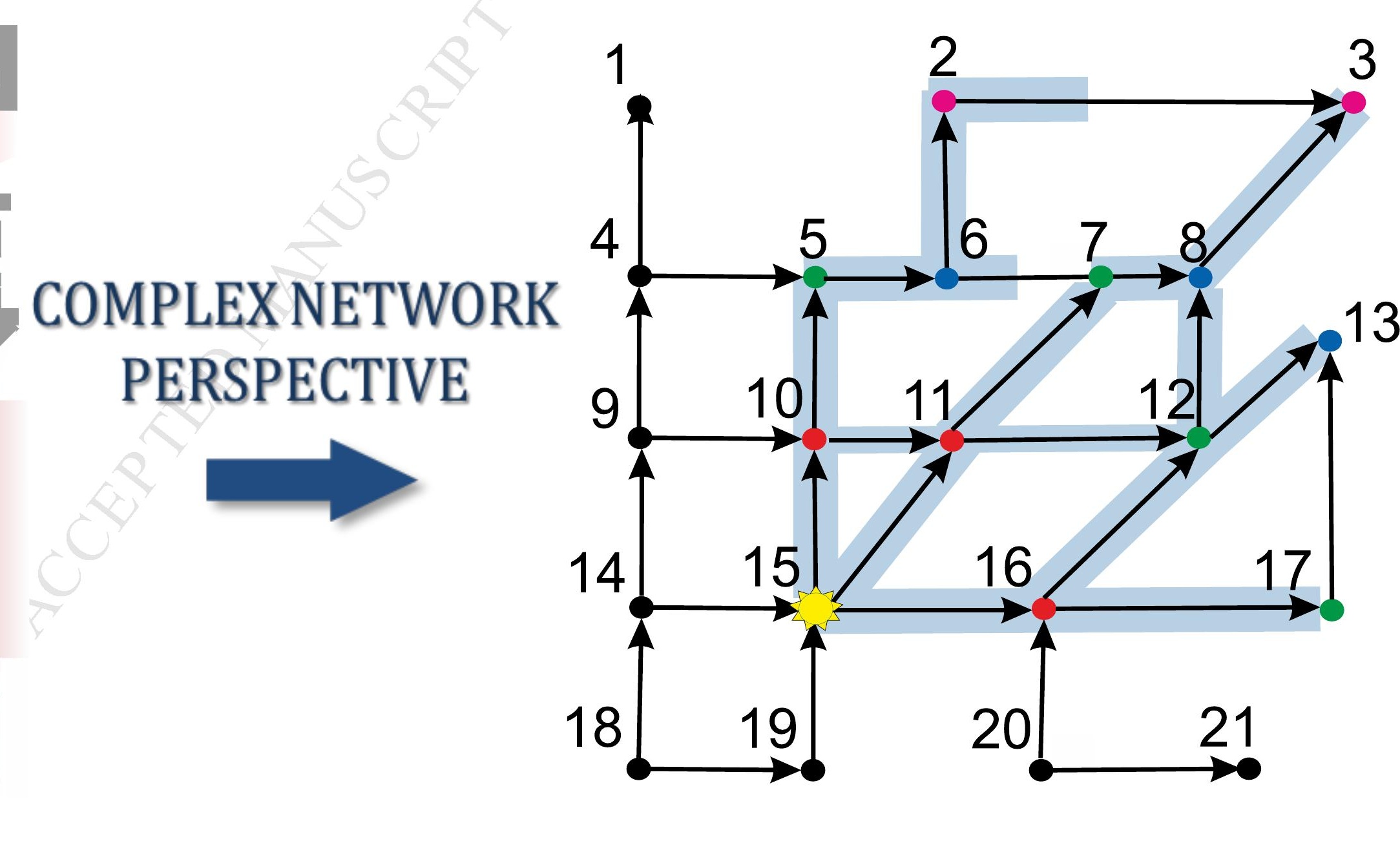




\section{Propagation of toxic substances in the urban atmosphere: a complex network perspective}

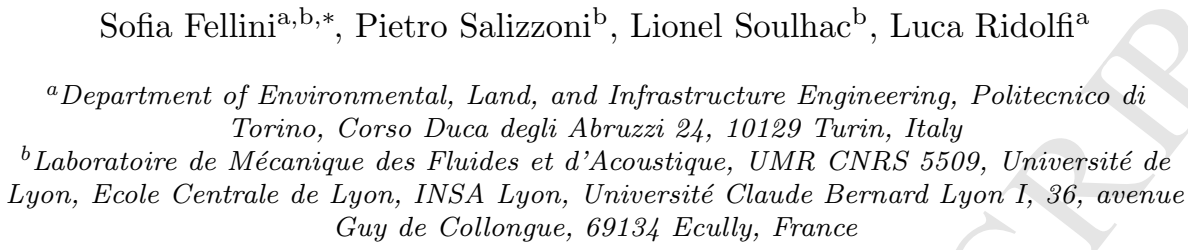

\section{Abstract}

The accidental or malicious release of toxic substances in the urban atmosphere is a major environmental and safety problem, especially in large cities. Computational fluid dynamics codes and simplified modelling tools have been used in the last decades to model pollutant dispersion in urban areas. These studies have shown that propagation is strongly influenced by the layout of buildings and, therefore, by the street topology of the city. This work presents a novel approach to the study of toxic propagation within the urban canopy based on the theory of complex networks. The urban canopy is modelled as a network, where the streets and the street intersections represent respectively the links and the nodes of the network. The direction and the weights of the links contain the geometrical characteristics of the street canyons and their wind conditions, so that all the key variables involved in pollutant dispersion are represented in a single mathematical structure that is a weighted and directed complex network. Thanks to this mathematical interpretation, propagation is modelled as a spreading process on a network and a depth-first search algorithm is used to rapidly delimit the zone of influence of a source node. This zone is the set of streets that are contaminated from the source. As a case study, the proposed model is applied to the urban tissue of the city of Lyon. The algorithm simulates a toxic release in all the nodes of the network and computes the number of people affected by each propagation process. In this way, the nodes with

${ }^{*}$ Corresponding author 
the most dangerous spreading potential are identified and vulnerability maps of the city are constructed. Moreover, various wind and concentration scenarios are easily implemented. The results highlight how the proposed method is effective in assessing the most vulnerable points in a city with a computational time that is up to three orders of magnitude lower than that of existing models. Moreover, the proposed approach paves the way to future applications of tools and metrics from the complex network theory for a deeper comprehension of the mechanisms that drive pollutant dispersion in urban areas.

Keywords: Urban air pollution, Street network, Complex networks, Vulnerability, Accidental releases, Spreading on networks

\section{Introduction}

Large cities are particularly vulnerable to air pollution as they exhibit both a large number of potential sources and a high density of people exposed (Brunekreef and Holgate, 2002; Heinrich and Wichmann, 2004). Urban air pollution is mainly linked to human activities and in particular to vehicular traffic, heating of buildings and industrial emissions (Mayer, 1999). Moreover, accidental releases such those related to gas leaks, industrial plants or the transport of dangerous goods are particularly critical in densely populated environments. Besides unintentional releases, the current political situation also raises the possibility of terrorist attacks aimed at the dispersal of toxic or pathogenic substances in the air (Tucker, 2000; McLeish, 2017).

For these reasons, local authorities are urged to adopt not only instruments for air quality control but also predictive tools for the management of dangerous situations due to accidental and malicious releases. These actions are in line with the current challenge of building resilient metropolises able to cope with emergencies (Berke et al. 2009; Ahern, 2011).

Dispersion models are commonly used to predict urban pollution and to estimate concentration of toxic substances. Computational fluid dynamics (CFD) simulations are the most accurate tool for modelling dispersion in a complex 
geometry like the urban fabric, since they solve the velocity and the concentration fields in the whole domain (Blocken, 2015). However, these models require a huge computational cost and therefore long simulation times and high performance computers. To reduce the computational cost, several modelling approaches have been developed in the last decades (Di Sabatino et al., 2013). These include street network models (e.g., Carruthers et al., 2000, Soulhac et al. 2011) based on a simplified description of the building geometry and modelling the mass exchange within and above the urban canopy by parametrising a few key transfer processes. Their formulation rely on the basic idea that the urban structure of the city, the orientation of the streets and their connectivity play a major role in determining the intensity of these transfer processes. The street network approach was originally proposed by Soulhac (2000), who developed the operational model SIRANE (Soulhac et al., 2011). Recently, Kim et al. (2018) presented a multi-scale model of urban air pollution that combines SIRANE with a comprehensive treatment of atmospheric chemistry. On the other hand, several efforts have been devoted to further simplify the model. Hamlyn et al. (2007) constructed a simple street network model for the dispersion of pollutants within a regular array of cubic obstacles. Belcher et al. (2015) and Goulart et al. (2018) derived an analytical formulation for atmospheric dispersion in a regular geometry and showed that the process can be described by basic geometrical and flow properties. Tzella and Vanneste (2016) developed a large deviation theory for concentration prediction in a rectangular network. These studies have highlighted the need for viable models able to capture the fundamental aspects of pollutant dispersion within urban-like geometries, in order to give quantitative estimates of mean concentration with a reduced computational cost and starting from few input data.

In this framework, we introduce a novel approach for the assessment of the vulnerability of a dense city to the release of harmful airborne pollutants. Going beyond the representation of the urban spatial domain as a street network, in this work all the key variables involved in the dispersion process are viewed as properties of a complex network. Thanks to this mathematical interpretation, 
the modern techniques of the theory of complex networks can be adopted and propagation phenomena in the streets are modelled as a transport process on a network.

In complex network theory (e.g., Boccaletti et al., 2006, Newman, 2010), complex systems are traced back to a set of entities (nodes) that interact with each other. Interactions are represented as links between the nodes and may have weights that describe the strength of these interactions. Complexity does not lie in the elements that form the system, but rather in their topology and within the pattern of their interconnections. In the last few years, the theory of complex networks has gained a great deal of attention in countless fields, from the social sciences (e.g., Borgatti et al., 2009) to engineering (e.g., Carvalho et al., 2009, Yazdani and Jeffrey, 2011, Giustolisi and Ridolfi, 2014). Recently, a network approach has been adopted for the description of geophysical fluid motion and associated transport phenomena. Gelbrecht et al. (2017) have proposed a complex network representation of wind flows to study regional meteorology systems, while Ser-Giacomi et al. (2015) have represented mixing and dispersion processes in the Mediterranean as a transportation network.

In this work we focus on the pollutant dispersion at the local urban scale. The topology of the city is modelled as a network, within which the propagation of the airborne toxic pollutant occurs. The streets and the street intersections are respectively the links and the nodes of the network. The direction and the weight of the links contain the fluid-dynamical properties of the flow within the streets and the geometrical characteristics of the bordering buildings. In this way, the topology of the city and the wind conditions along the streets are all represented in a single mathematical structure, that is a weighted and directed complex network. Differently from street network models as SIRANE, a numerical solution of a system of mass balance equations is here not required. Given an initial point, i.e. a pollutant source, propagation along the streets is modelled as an epidemic on a network. In particular, as in epidemic applications (Newman, 2002), a search algorithm on networks is adopted to delimit the zone of influence of a source node, that we define as the part of the network affected 
by toxic propagation. By counting the number of people that reside in the zone of influence, a vulnerability index of the source node can be estimated. The procedure is applied to each node of the network and vulnerability maps are easily constructed, in order to reveal at a glance the urban areas with the highest spreading potential.

The method is much faster than previous models, and can be applied to entire cities with a low computational cost and with few input parameters. Given the simplified assumptions underlying the method, the results are not expected to be as accurate as the ones obtainable with more complete models. However, a very detailed estimate of pollutant concentration in every point of the city is generally not required for the assessment of urban vulnerability. The aim of this work is to provide a reliable and rapid technique to identify the most vulnerable points in the city, i.e. the source points from which the toxic spreading can affect the greatest number of people, and to understand the effect of meteorological conditions (namely wind direction) on urban vulnerability. Moreover, this work lays the foundation for further applications of tools from complex network theory. In particular, the new complex network perspective facilitates the investigation of the fundamental aspects that drive dispersion in the urban environment. In fact, the model outcome is not the overall result of a set of parametrizations and mass balance equations but is the result of a spreading process on a network characterized by well defined adjacency and weight matrices. Simple metrics (e.g., centrality metrics) and analyses from complex network theory can thus be applied to understand the role of the key variables (e.g., the topology of the city, the fluid-mechanical and geometrical characteristics of the streets) in the dispersion process.

The work is organized as follows. In Section 2, we describe the physical assumptions adopted to model pollutant propagation in the urban canopy. Then, the network perspective is introduced in Section 3 . The basic steps to construct the street network and its weight matrices are thus described and the algorithm for spreading on networks is presented. Subsequently, the proposed general method is applied to the city of Lyon (France). Vulnerability maps 
for a district of Lyon are shown and analysed in Section 4 . Finally, the main conclusions obtained from the present work are summarized.

\section{Physical assumptions about the propagation of a toxic substance in the urban environment}

Transport and mixing processes in the urban environment are characterized by complex fluid structures due to the interaction between the atmospheric flow and the city. The presence of buildings and vegetation highly affects the structure of the urban boundary layer, characterized by the generation of a shear layer at the top of the canopy, wake diffusion behind buildings, and form drag due to the pressure differences across the roughness elements (Roth, 2000). Moreover, the flow field in the streets is altered by the convective fluxes due to the differential solar irradiance on building walls and to the heat sources related to human activities (Oke, 1982; Arnfield, 2003). The simulation of all these dynamical effects on the flow field within the urban canopy is nowadays a challenge for modellers that adopt sophisticated computational tools, typically CFD codes (Tominaga and Stathopoulos, 2012). These require a huge amount of input data and high computational costs, which limit their use when analysing a large number of emission scenarios. In this latter case, alternative simulation strategies should be adopted, based on a simplified description of the flow and of the dispersion phenomena occurring within the urban area.

The physical assumptions for the propagation model presented in this work are inspired by the street network model approach (e.g., Namdeo and Colls, 1996 Soulhac et al. 2011). In street network models, the geometry of the urban canopy is simplified to a network of streets (Fig. 1.a), and the streets are represented as urban canyons, i.e. cavities of rectangular section with length $l$, height $h$ and width $w$ (Fig. 11.b). Following the approach of Soulhac et al. (2011), the main transport phenomena for an airborne pollutant in the urban canopy are (i) the advective mass transfer along the street due to the mean wind along the longitudinal axis, (ii) the vertical transfer from/toward the external 
atmosphere and (iii) the transport at street intersections. In street network models, pollutant dispersion is then described by a series of mass balance equations within streets, at street intersections and above the urban canopy. This system of equations is numerically solved to evaluate pollutant concentrations within the streets and above them.

To assess the vulnerability of urban areas to toxic releases, a detailed quantification of the concentration in the streets and in the street intersections is generally not required. Rather, an operational model based on few input data and with very low computational cost is sought, so that it can be easily and quickly implemented for the assessment of large city vulnerability, even in emergency situations. For these reasons, numerical solution of mass balance equations and the estimation of a large number of physical parameters are here avoided. Conversely, we introduce an analytical solution for dispersion along a street canyon, we adopt a simplified model for propagation in street intersections and we use concepts from complex network theory to simulate toxic spreading from one street to the adjacent ones. To this aim, further simplifications are required compared to those adopted in well-known street network models (e.g., Soulhac et al. 2011). These simplifications concern the physico-chemical processes of pollutants in the street canyons, vertical transfers between the canyon and the atmosphere, and pollutant fluxes in the intersections.

In the following, the transport mechanisms along a street canyon and at street intersections are described in detail. The adopted physical assumptions are introduced and discussed. The emission scenario consists of a release from a point source ( $s$ in Fig. 1) at ground level within the urban canopy. The external wind blowing on the city with direction $\Phi$ is the driving force for propagation processes.

\subsection{Transport along a street canyon}

Consider a source $s$ releasing a gaseous substance that results in a concentration $c_{0}$ at the beginning of a street canyon, as illustrated in Fig. 11.b-c. The main transport processes are the advective flux along the longitudinal axis, 
a)

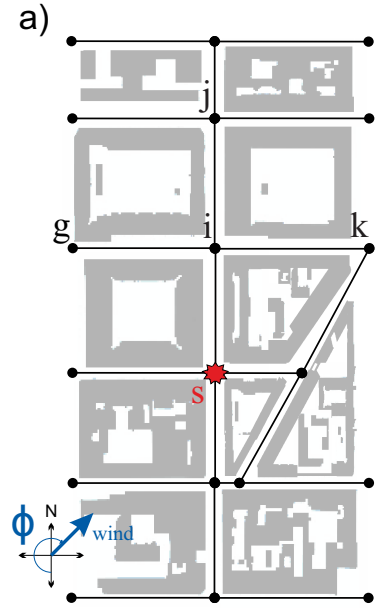

b)

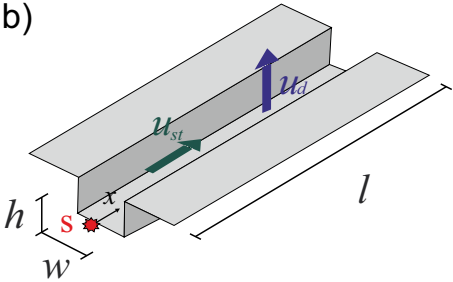

c)

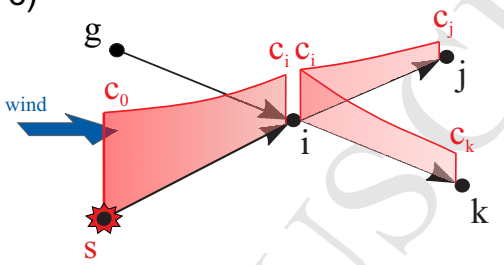

Figure 1: a) Toxic source $s$ within a network of streets. Propagation is driven by the wind blowing on the city with direction $\Phi$. b) Representation of a street canyon with the main variables of the model. c) Transport of a contaminated flow in street intersections.

and the vertical turbulent exchanges between the street and the overlying atmosphere (Soulhac et al. 2013). In fact, turbulent longitudinal diffusion is marginal compared to the longitudinal advection, while physico-chemical processes are generally negligible as their typical time scales are longer than the time needed for propagation. Under these assumptions, the transport along a street canyon can be modelled with the one-dimensional transport equation

$$
\frac{\partial c}{\partial t}+u_{s t} \frac{\partial c}{\partial x}+\frac{u_{d}}{h}\left(c-c_{e x t}\right)=0
$$

whose solution is the function $c(x, t)$, i.e. the concentration along the longitudinal coordinate $x$ over time $t$.

The first two terms in (1) describe the advective transport driven by the spatially averaged wind velocity along $x\left(u_{s t}\right)$. This velocity is assumed to be given by a balance between the stress imposed at the canopy top by the external atmospheric flow and the drag due to the roughness of the canyon walls (neglecting the role of pressure gradients). Under this assumption, Soulhac et al. (2008) derived an analytical formulation for $u_{s t}$, as a function of the external wind intensity and direction, the geometry of the street canyon and the 
aerodynamic roughness of building walls (see Appendix A. The third term in (1) models the mass transfer from/to the canyon to/from the overlying atmosphere by means of a bulk exchange velocity $u_{d}$ (Salizzoni et al., 2009, Soulhac et al. 2011) estimated as

$$
u_{d}=\frac{u_{*}}{\sqrt{2} \pi},
$$

where $u_{*}$ is the friction velocity of the overlying boundary layer flow. We underline that the specific physical models adopted here for $u_{s t r}$ and $u_{d}$ could be easily replaced if better ones become available. Moreover, a simple model for physico-chemical transformation could be easily implemented in (1) by adding a physico-chemical transformation rate to the exchange velocity $u_{d}$.

Since our focus is on a localized ground-level release only - therefore inducing maximal concentration at the street level -, we will assume that the vertical flux of pollutants from the canopy to the external atmosphere will induce significantly low concentrations, $c_{e x t}$, above roof level, due to the high dilution occurring in the lower part of the boundary layer compared to that within the streets. As a consequence, we will consider that $c-c_{e x t} \simeq c$, therefore ignoring the role of any re-entrainment of mass from the external flow to the streets. The effects of re-entrainment have been discussed by different authors (Hamlyn et al., 2007, Belcher et al., 2015; Goulart et al., 2018). Their studies highlighted that re-entrainment effects are physically significant in the so called far-field region, i.e. the region extending from the third intersection downwind the source. However, the vertical flux is in general less than a quarter of the horizontal advective flux (Goulart et al., 2018). For these reasons, the possible inclusion of re-entrainment effects in the model is not expected to bring significant improvements to the results.

Equation (1) was analytically solved for both an instantaneous and a continuous release in the source (see Appendix B. In the first case, the initial condition is set as a rectangular pulse with height $c_{0}$. The solution describes this initial step travelling along the street with velocity $u_{s t}$ and undergoing an 
exponential decay of concentration. In the second case, the initial condition is a continuous release with constant concentration $c_{0}$. The solution is a front that spreads along the street with velocity $u_{s t}$. Although the analytical solutions are different, the concentration at the end of the street $(x=l)$ in both cases is the same:

$$
c_{l}\left(x=l, t=\frac{l}{u_{s t}}\right)=c_{0} e^{-\frac{l}{u_{s t}} \frac{u_{d}}{h}} .
$$

Thus, the concentration at the beginning of the street $\left(c_{0}\right)$ undergoes an exponential decay driven by the ratio between the advection time $\left(l / u_{s t}\right)$ that the toxic front spends to reach the end of the street, and the vertical transfer time $\left(h / u_{d}\right)$. The terms in the exponent summarize all the information about the geometry of the canyon and the flow dynamics in it.

\subsection{Transport in the street intersections}

The flow field in the street intersections is driven by complex physical processes that depend on multiple geometrical and meteorological parameters. Several studies (e.g. Hunter et al., 1990, Robins et al., 2002, Soulhac et al., 2009) have demonstrated that even slight variations in the building geometry and wind direction can affect significantly the redistribution of the incoming fluxes over the outgoing fluxes. On the basis of these observations, Soulhac et al. (2009) have developed a model, quantifying the balance of the time-averaged incoming and outgoing fluxes at the street intersection. This model requires the estimation of exchange coefficients between the streets, and the formulation of a mass balance equation for each intersection. In street network models (e.g., SIRANE), these balance equations are solved numerically together with the equations for transport along the canyons.

As mentioned in Section 2 our aim is to propose a simplified methodology for pollutant dispersion modelling, that simulates propagation as a spreading process on a network. In order to adopt this approach, an analytical solution for the physical transport mechanisms is required. For these reasons, we define 
a simple exchange model in the intersections that avoid the use of mass balance equations. Given the uncertainty of flow redistribution in street intersections, a conservative approach is adopted in developing this minimalistic model.

Consider a simple intersection with a single incoming and one outgoing street canyon. The volumetric air flow rate entering the intersection with a concentration $c_{i n}$ is $Q_{i n}$, while the volumetric air flow rate outgoing the intersection with a concentration $c_{\text {out }}$ is $Q_{\text {out }}$. Two cases are possible for the mass balance in the intersection. In the first case, the mass flow rate from the incoming canyon $\left(\dot{m}_{i n}=c_{i n} Q_{i n}\right)$ is lower than the mass flow rate toward the outgoing canyon $\left(\dot{m}_{\text {out }}=c_{\text {out }} Q_{\text {out }}\right)$. In order for the mass balance to be satisfied, an external mass flow rate $\left(\dot{m}_{\text {ext }}\right)$ from the atmosphere above the canopy enters the intersection vertically with a volumetric air flow rate $Q_{e x t}$ and a concentration $c_{e x t}$, so that

$$
\dot{m}_{i n}+\dot{m}_{\text {ext }}=\dot{m}_{\text {out }} \rightarrow c_{\text {in }} Q_{\text {in }}+c_{\text {ext }} Q_{\text {ext }}=c_{\text {out }} Q_{\text {out }} .
$$

In Section 2.1, we stated that re-entrainment from the atmosphere is negligible in our model. Thus, the mass flow rate from the atmosphere makes a zero contribution in (4). The concentration $c_{\text {out }}$ at the beginning of the street outgoing the intersection is therefore given by $c_{\text {out }}=c_{i n} Q_{\text {in }} / Q_{\text {out }}$. As the volumetric flow rate balance in the intersection is $Q_{\text {in }}+Q_{\text {ext }}=Q_{\text {out }}$, the ratio $Q_{\text {in }} / Q_{\text {out }}$ is lower than 1 and thus $c_{\text {out }}<c_{\text {in }}$. In the second case, $\dot{m}_{\text {in }}>\dot{m}_{\text {out }}$ and the mass flow rate $\dot{m}_{\text {ext }}$ leaves the intersection vertically, in order for the mass balance to be satisfied:

$$
\dot{m}_{\text {in }}=\dot{m}_{\text {ext }}+\dot{m}_{\text {out }} \rightarrow c_{\text {in }} Q_{\text {in }}=c_{\text {ext }} Q_{\text {ext }}+c_{\text {out }} Q_{\text {out }}
$$

We consider that the concentration leaving the intersection is the same for both the upwards flow and the flow towards the outgoing street canyon, i .e. $c_{\text {ext }}=c_{\text {out }}$. Applying (5) and the volumetric flow rate balance equation $\left(Q_{\text {in }}=\right.$ $\left.Q_{\text {out }}+Q_{\text {ext }}\right)$, we find that $c_{\text {out }}=c_{\text {in }}$.

These arguments show that in the case of an intersection with a single in- 
coming and one outgoing street canyon, the concentration $c_{\text {out }}$ is equal or lower than the concentration $c_{i n}$. To maintain a conservative approach, in our model we assume that the concentration of pollutants at the beginning of the street outgoing the intersection is the one at the end of the incoming contaminated canyon.

In the case of several streets crossing, we assume that when a contaminated puff with concentration $c_{i n}$ reaches the intersection, then the concentration at the beginning of the streets exiting the intersection is $c_{i n}$. According to this assumption, we consider that when a contaminated flow reaches the intersection, it does not divide between the different outgoing streets but tends to spread towards one of them. Given the uncertainty associated with the trajectory taken by the pollutant puff (Scaperdas, 2000), we consider all the possible statistical realizations, and thus we assume that all the outgoing streets are contaminated by the incoming concentration $c_{i n}$.

The assumptions adopted for the transport in street intersections are clearly illustrated in Fig 1. The contaminated flow from street $(s, i)$ propagates towards streets $(i, j)$ and $(i, k)$. According to this scheme, the concentration at the beginning of streets $(i, j)$ and $(i, k)$ is the one at the end of street $(s, i)$.

\section{A network perspective}

In the model presented above, toxic substances move in the urban environment driven by the wind blowing along the street canyons. The street canyons behave like upward leaking transport channels and their geometry, position and connectivity strongly influence the propagation. In big cities, streets cross each other to compose intricate patterns (Fig. 2). Given the spatial extent and the high number of elements, these urban fabrics can be seen as complex networks (Porta et al., 2006, Barthélemy, 2011). Links stand for the street canyons, while nodes represent the street intersections. The direction and the weight of the links describe the geometrical and fluid-dynamical properties of the street canyons. Within this approach, the tools of network theory provide interesting 
information about the propagation phenomena.

\subsection{Construction of the network}

The urban canopy is modelled as a network of $N$ nodes (intersections) and $M$ links (streets). Fictitious nodes can be created to divide a street into two links in case there is a significant change in the street properties. Each link is directed according to the orientation of the mean wind along the street $\left(u_{s t}\right)$. Thus, the network structure represents both the topological properties of the urban fabric and the directions in which the propagation processes take place. The connectivity of the street canyons is described by the adjacency matrix $\mathbf{A}$, a $N \times N$ square matrix whose element $A_{i j}$ is equal to 1 if a directed link from node $i$ to node $j$ exists, is equal to 0 otherwise (see Appendix C for an example of adjacency matrix). Since the links have a specific direction, the adjacency matrix is asymmetric.

According to this network representation, the geometry and fluid-dynamical properties of the street canyons are stored efficiently in matrices. $\mathbf{L}$ and $\mathbf{H}$ are the symmetrical matrices of the length of the streets $(l)$ and the average height of the buildings overlooking the streets $(h)$. The wind velocity along the streets $\left(u_{s t}\right)$ and the velocity of the vertical transfer towards the external atmosphere $\left(u_{d}\right)$ are enclosed in the matrices $\mathbf{U}$ and $\mathbf{U}_{\mathbf{d}}$.

As mentioned in the Introduction, the main purpose of this work is to establish a methodology for the rapid assessment of urban vulnerability to the ground-level release of toxic gases. The vulnerability index $\left(V_{s}\right)$ for a generic node $s$ can be defined as the number of people affected by the toxic propagation if the release takes place in $s$. The adopted network approach and matrix notation make it easy to calculate $V_{s}$ as follows,

$$
V_{s}=\sum_{i}^{N} \sum_{j}^{N} D_{i j} P_{i j},
$$

where $\mathbf{P}$ is the matrix that associates at each link $(i, j)$ the number of inhabitants per unit street length and $\mathbf{D}$ is the matrix that associates at each link $(i, j)$ 

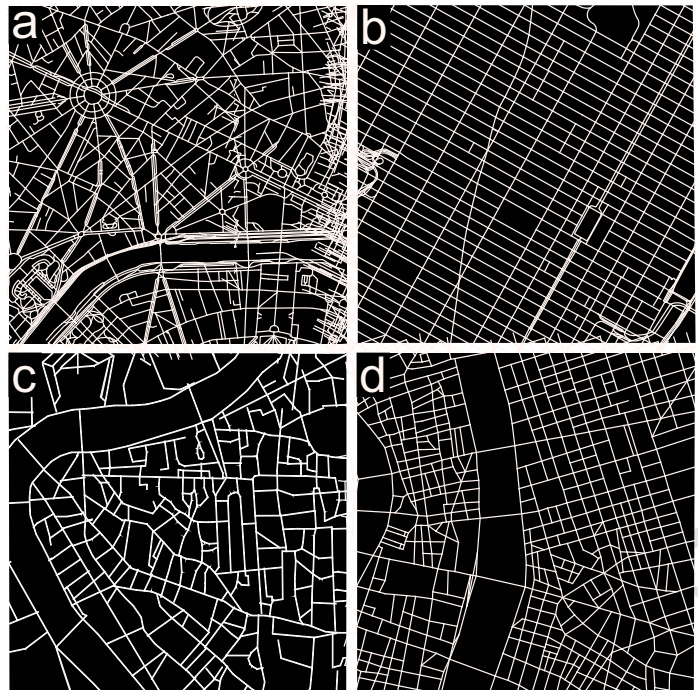

Figure 2: Snapshot of the street network of Paris (a), New York (b), Rome (c), and Lyon (d).

the contaminated length of the street. This last matrix represents the zone of influence of the source node. The meaning of this matrix and its construction process will be widely described in Subsections 3.2 and 3.3

An interesting advantage of this compact notation is that changes to the network properties can be easily implemented. By modifying matrices $\mathbf{U}$ and $\mathbf{U}_{\mathbf{d}}$, we can simulate different meteorological scenarios, while variations in population distribution (e.g., differences between weekdays and holidays) can be considered by adjusting $\mathbf{P}$. Furthermore, new buildings and structural changes are included in the model by revising the single elements of $\mathbf{L}$ and $\mathbf{H}$.

\subsection{Propagation of a toxic substance in the street network}

Consider the release of a toxic substance in a street intersection (see Fig. 3a). According to the hypotheses of our model (Section 2), (i) the substance propagates along the adjacent streets depending on the direction of the wind, (ii) the concentration decays exponentially along the streets, (iii) the concentration at the end of each street can be estimated using (3), (iv) the concentration remains unchanged in the street intersections, and (v) from a contaminated street intersection the gas spreads further to the adjacent streets. 
Given this description, the pollutant dispersion within the canopy can be then easily seen as a spreading process on a network (e.g., Newman, 2002 , Comin and da Fontoura Costa, 2011). In Fig. 3 b the urban canopy is represented as a network. The links are directed according to the direction of the wind in the streets and the release is modelled as a source node $s$. From a network perspective, transport from $s$ towards a generic node $u$ is possible if there is a link directed from $s$ to $u$, i.e. if (i) the two nodes are physically connected by a street canyon, and (ii) the wind is blowing from the source towards the target node. Once infected, the target node $u$ is modelled as a new source and the spreading process carries on towards the farthest nodes. According to this scheme, propagation is a recursive process that spontaneously expands to the topological boundaries of the network. Physically, the extent of the contaminated zone can be delimited based on a threshold concentration value $c_{t h}$ : when the concentration falls below $c_{t h}$, the contamination process is irrelevant. Thus, a stopping rule for the spreading process on the network is introduced: at each propagation hop the concentration at the target node is estimated. If this concentration is higher than $c_{t h}$, then the propagation carries on.

Considering the example in Fig. 3. b, a toxic substance is released in the source node $s=15$ and propagates towards the first neighbours of $s$ : nodes 10, 11, and 16. The concentration in the first neighbours is evaluated using Equation (3), as a function of the geometrical and wind characteristics of the street canyons associated to links $(15,10),(15,11)$ and $(15,16)$. Since the concentration in the first neighbours is grater than the predefined threshold $c_{t h}$, nodes 10,11 and 16 act as source nodes and the spreading process carries on towards the second neighbours of $s$. In the same way, the third and fourth neighbours are affected by the toxic propagation until the concentration in the nodes falls below $c_{t h}$.

According to this network interpretation, the spreading of a toxic gas in the urban environment is governed by two properties of the network: (i) the topological connectivity of the network, given by its adjacency matrix $\mathbf{A}$, and (ii) the concentration decay along the links, given by a combination of the 
a)

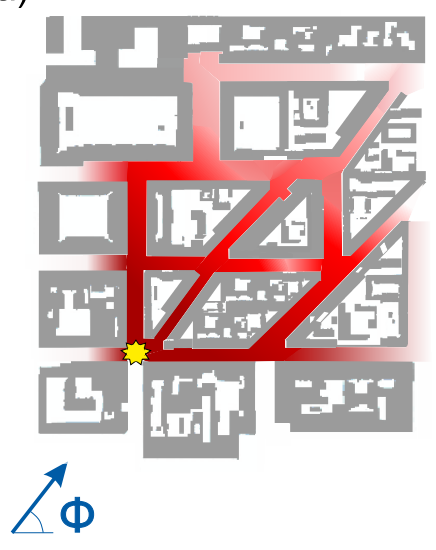

b)

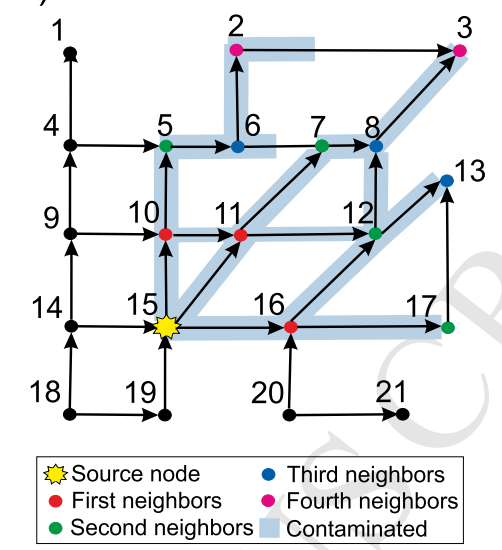

Figure 3: Analogy between the physical propagation of a toxic substance in the urban environment (a) and the spreading process on a network (b). $\Phi$ is the direction of the external wind blowing on the city.

geometrical characteristics and the flow dynamics in the street canyons.

This spreading results in the zone of influence of the source node $s$, i.e. the set of links contaminated from the propagation process originated in $s$ (the elements highlighted in blue in Fig. 33. The number of people living in the zone of influence represents the vulnerability index of $s\left(V_{s}\right)$. Notice that this index contains both information on population density and meteorological conditions in the city. In fact, as will be shown in the following sections, its value changes drastically with the wind direction.

\subsection{Algorithm}

Given a source node $s$ with a concentration $c_{s}$, the set of its first neighbours $N_{1}(s)$ can be derived from the non-zero elements of the $s-t h$ row of the adjacency matrix A. In Fig. $3, s=15$ and thus $N_{1}(s)=\{10,11,16\}$. For each node $u$ belonging to the set $N_{1}(s)$, the algorithm calculates the distance $D_{s u}^{p o t}$. This length is the potential distance (hence the superscript pot) that the contaminated front can reach along the link $(s, u)$ with a concentration higher 
than the predefined threshold $c_{t h}$. According to (3), $D_{s u}^{p o t}$ is

$$
D_{s u}^{p o t}=-\frac{H_{s u}}{U_{d, s u}} U_{s u} \log \left(\frac{c_{t h}}{c_{s}}\right), \quad \forall u \in N_{1}(s) .
$$

In general, $D_{s u}^{p o t}$ is different from the physical length of the street $\left(L_{s u}\right)$ associated to the link $(s, u)$. $D_{s u}^{p o t}$ is lower than $L_{s u}$ if the concentration undergoes the threshold $c_{t h}$ before the propagation front has travelled the entire street. Conversely, it is higher if the front reaches node $u$ with a concentration above the threshold. As a consequence, the effective contaminated distance $\left(D_{s u}\right)$ is the minimum between the reachable distance $D_{s u}^{p o t}$ and the effective length of the street, i.e.

$$
D_{s u}=\min \left[D_{s u}^{p o t}, L_{s u}\right]
$$

If $D_{s u}=L_{s u}$, the substance has reached the target node $u$ with a concentration equal to or higher than $c_{t h}$. As a result, node $u$ is contaminated. Conversely, if the front reaches $u$ with a negligible concentration (i.e. $D_{s u}<L_{s u}$ ), then node $u$ remains uncontaminated. In both cases, the algorithm stores the effective contaminated length $D_{s u}$ as the $(s, u)$ element in the matrix D. As introduced in Section 3.1 this matrix defines the zone of influence of the source node $s$.

We define $\hat{N}_{1}(s)$ the set of the first neighbours of $s$ that have been contaminated,

$$
\hat{N}_{1}(s)=\left\{u \in N_{1}(s) \mid D_{s u}=L_{s u}\right\}
$$

Referring to Fig. 3. $\hat{N}_{1}(15)=\{10,11,16\}$ since all the first neighbours of 444 are contaminated. The algorithm estimates the concentration in the nodes belonging to $\hat{N}_{1}(s)$ using $\sqrt{3}$, as

$$
c_{u}=c_{s} e^{-\frac{U_{d, s u}}{H_{s u}} \frac{L_{s u}}{U_{s u}}}, \quad \forall u \in \hat{N}_{1}(s) .
$$


These nodes behave as new source nodes. Thus, the algorithm repeats the above presented steps, replacing in Equations (7)-10 node $s$ with the nodes belonging to $\hat{N}_{1}(s)$. For example, once node 16 (Fig. 3) has been contaminated from the initial source node 15, the algorithm finds the set of its first neighbours, i.e. $N_{1}(16)=\{12,17\}$. Equations (7)-8 estimate the effective contaminated length along links $(16,12)$ and $(16,17)$, while $(9)$ identifies the set of the first neighbours of node 16 that have been infected, i.e. $\hat{N}_{1}(16)=\{12,17\}$. Finally, the concentration reached in nodes 12 and 17 is determined by $(10)$. This procedure is repeated recursively until the concentration in each node of the network falls below the threshold $c_{t h}$.

Notice that for nodes 15 and 16 the sets $N_{1}$ and $\hat{N}_{1}$ are identical. However, this is not true in general. Consider node 6 in Fig. 3b. The set of its first neighbours is $N_{1}(6)=\{2,7\}$, while $\hat{N}_{1}(6)=\{2\}$ because node 7 cannot be reached by the propagation along link $(6,7)$.

The algorithm explores the nodes of the network starting from a root node and progressively visiting the adjacent nodes. In computer science, this process is called tree traversal (Valiente, 2013) since the result of the exploration is a tree structure that is a subgraph of the initial graph. In our study, this tree corresponds to the zone of influence of the source node. There are multiple ways to perform a tree traversal, according to the order in which the nodes are visited. The algorithm we use explores the nodes of the network using a depth-first search analysis. The algorithm starts at the source node and goes as far as it can down a given branch before backtracking. Referring to Fig. 3 . the algorithm starts at node 15 , selects the first node 10 in the set $\hat{N}_{1}(15)$ and deepens the analysis in the first element of the set $\hat{N}_{1}(10)$, i.e. node 5 . Following this method, the algorithm visits the nodes in the order $(15,10,5,6,2)$. The in-depth analysis along this path ends when the concentration falls below the threshold $c_{t h}$. Formally, the path $(15,10,5,6,2)$ ends because the set $\hat{N}_{1}(2)$ is empty. Once the first branch has been explored, the algorithm backtracks to node 6's next available neighbour, i.e. node 7 . 
From the numerical point of view, depth-first search analysis requires less memory and it is more efficient in finding trees on networks compared to other algorithms, such as breath-first search (Kozen, 1992).

Notice that, referring to the example in Fig. 3b, link $(11,7)$ is affected by toxic propagation twice, both along propagation path $\gamma_{1}=(15,11,7)$ and $\gamma_{2}=(15,10,11,7)$. As a consequence the algorithm calculates two different values of $D_{11,7}$, since the concentration reached at node $11\left(c_{11}\right)$ along $\gamma_{1}$ is generally different from the one obtained along $\gamma_{2}$. As the aim of the method is to determine the extent of the zone of influence, the algorithm considers all the possible passages through a generic link and stores the longest distance reached by the toxic substance. More precisely, for each path $\gamma_{\alpha}$ the algorithm compares $D_{s u}^{\left(\gamma_{\alpha}\right)}$ with the distance $D_{s u}^{\left(\gamma_{\alpha-1}\right)}$ obtained along the previously explored path $\gamma_{\alpha-1}$ passing through the link $(s, u)$. Equation $(8)$ is, thus, refined as:

$$
D_{s u}^{\left(\gamma_{\alpha}\right)}=\max \left[\min \left[D_{s u}^{p o t}, L_{s u}\right], D_{s u}^{\left(\gamma_{\alpha-1}\right)}\right]
$$

where $\gamma_{\alpha}$ is an index for the order in which the path is explored. We underline that, given the assumptions in Subsection 2.2, the two contaminated flows along paths $\gamma_{1}$ and $\gamma_{2}$ do not physically meet in node 11. Rather, $\gamma_{1}$ and $\gamma_{2}$ are two of the possible contaminated paths originated by the pollutant emission.

\section{Results}

The potential of the proposed approach is discussed through a case study. The model is applied to assess urban vulnerability of the city of Lyon (France) to the release of a toxic gas. Lyon (Fig. 4 ) is located in east-central France and it is the third-largest urban agglomeration in France after Paris and Marseille, with a population of approximately 1.5 million inhabitants. In this work, the analysis is limited to a part of the city (Fig. 4r) that presents an intricate urban fabric and tall buildings on the edge of the streets. These characteristics are consistent with the model representation of the streets as a network of street canyons. The study area has an extent of about $6.5 \mathrm{~km}^{2}$, it hosts a population of 


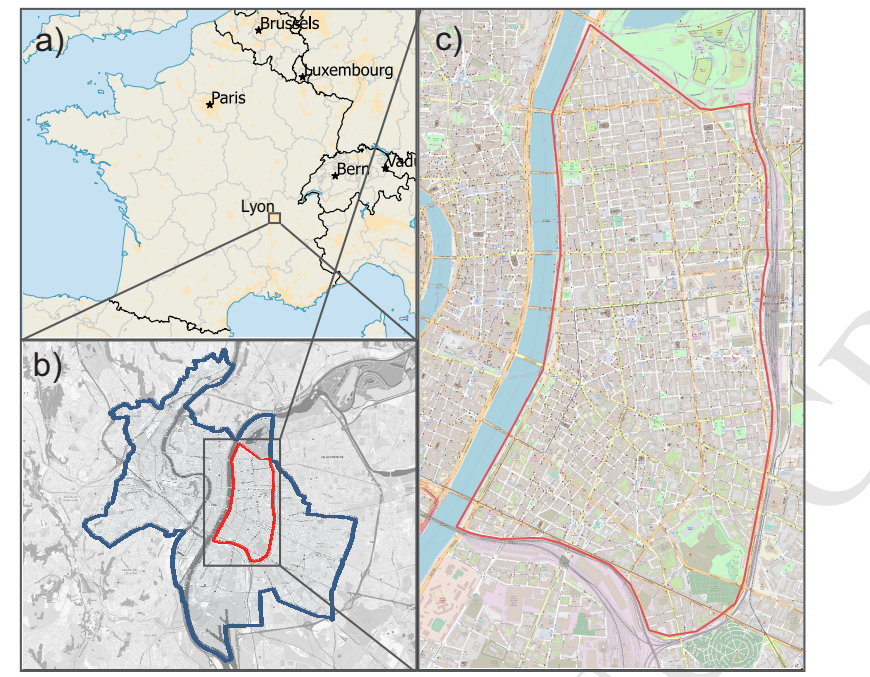

Figure 4: The case study area. a) Location of the metropolitan area of Lyon within France. b) Location of the study area within the municipality of Lyon. c) The study area.

about 140,000 inhabitants and is delimited by natural and artificial boundaries (rivers, parks and railways). These boundaries determine a discontinuity in the dispersion of pollutants along the street canyons.

The street canyons and the street intersections result in a network of 750 nodes and 1110 links. The geometrical characteristics of the street canyons, the population density, the longitudinal mean wind and the vertical transfer velocity in the streets are stored in the matrices $\mathbf{H}, \mathbf{L}, \mathbf{P}, \mathbf{U}$ and $\mathbf{U}_{\mathbf{d}}$, respectively.

\subsection{The zone of influence of a source node}

The first outcome of the model is the identification of the zone of influence of a source node, i.e. the set of links contaminated by the toxic substance with a concentration above a defined threshold, stored in matrix $\mathbf{D}$ (see Section 3 ).

As an example, Fig. 5 shows the zone of influence of a source node for different wind directions $\left(\Phi=45^{\circ}, 135^{\circ}, 225^{\circ}\right.$ and $\left.315^{\circ}\right)$ and for two different initial concentration values. For the sake of generality, the concentration scenarios are defined by the ratio between the concentration in the source node $\left(c_{0}\right)$ and the limit concentration $\left(c_{t h}\right)$. 

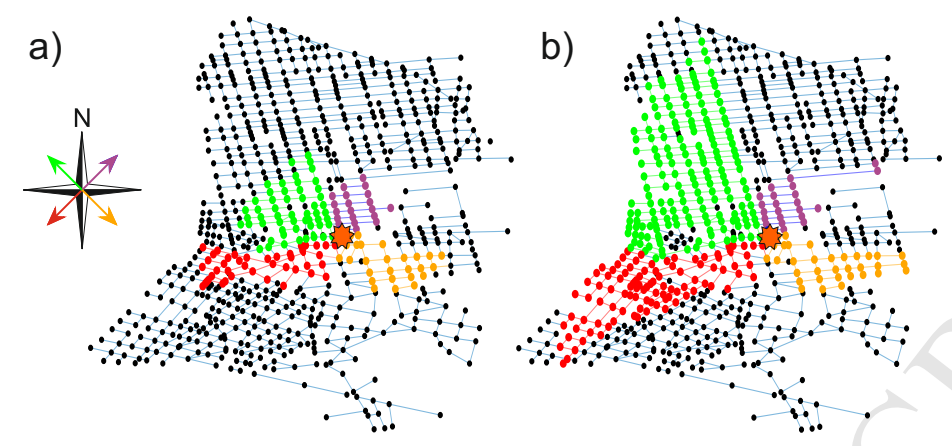

Figure 5: The zone of influence of a generic source node (orange star) in the network for different wind directions (red, green, violet and yellow refer to the wind directions $\Phi=45^{\circ}$, $135^{\circ}, 225^{\circ}$ and $315^{\circ}$, respectively). Panel (a) and (b) refer to a concentration ratio $c_{0} / c_{t h}=10$ and $c_{0} / c_{t h}=100$, respectively.

The urban topology, the wind direction and the initial concentration shape the zone of influence of the source node. Variations in the mean velocity of the external wind $(\bar{u})$ as well as the stability conditions (as determined by the Monin-Obukhov length $L_{M O}$ ) are instead irrelevant. As stated in (2) and (A.1)A.2), both $u_{d}$ and $u_{s t}$ depend linearly on the friction velocity $u_{*}$. Since our propagation model evaluates the pollutant spreading as a function of their ratio (3), variations of $u_{*}$, and thus of $L_{M O}$ and $\bar{u}$, will not be effective in determining the zone of influence.

\subsection{Spatial and frequency distribution of urban vulnerability}

The spatial pattern of urban vulnerability can be analysed at a glance using vulnerability maps. Given the geometry of the urban fabric, the conditions of the external wind, the spatial distribution of the citizens and the emission scenario, the model provides a map that associates at each node of the street network its vulnerability index. The computational time is 2 (in the case of $c_{0} / c_{t h}=100$ ) or 3 (in the case of $c_{0} / c_{t h}=10$ ) orders of magnitude lower than the time taken by well-known street network models as SIRANE. As mentioned in Section 3, this is achieved by first applying the algorithm for the spread of toxic substances on networks (Section 3) and by computing the matrix of the contaminated street lengths $\mathbf{D}$ (i.e. the zone of influence) for all nodes of the 


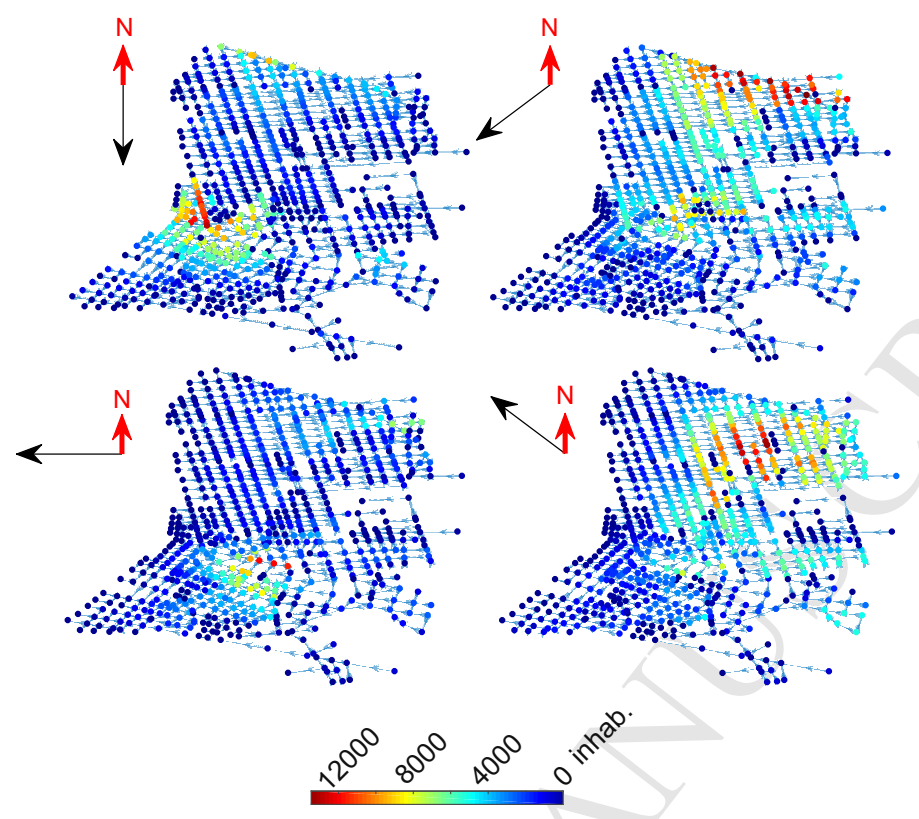

Figure 6: Vulnerability maps for different wind directions and with a concentration ratio $c_{0} / c_{t h}$ equal to 10 .

network. Next, the vulnerability index defined in Equation (6) is calculated for each node, taking into account both the extent of the zone affected by the propagation originated in the node $(\mathbf{D})$ and the number of people leaving in that zone $(\mathbf{P})$. Finally, a vulnerability map is constructed by associating a colour to each node based on its vulnerability index.

Sixteen vulnerability maps for the case study area are obtained (see the supplementary material) by varying the direction of the external wind $(\Phi)$ and the concentration ratio $c_{0} / c_{t h}$. These parameters affect the node vulnerability by shaping its zone of influence (matrix D), as mentioned in Section 4.1. In this study, the number of inhabitants in the streets $(\mathbf{P})$ was kept constant in the different scenarios and was derived from the map of the resident citizens in the city of Lyon. Future works should consider how the spatial distribution of the population varies on different days of the week or at different times of the day. These variations could be easily implemented in the model by modifying the $\mathbf{P}$ matrix and would increase the number of cases (i.e. maps) considered. 
Fig. 6 shows four vulnerability maps for a wind direction varying between $\Phi=0^{\circ}$ (north wind) and $\Phi=135^{\circ}$. The maps are obtained simulating for each node a release with a concentration equal to ten times the threshold value, i.e. $c_{0} / c_{t h}=10$. Node vulnerability, defined in terms of number of affected people, varies between 0 (blue) and 12000 (red) inhabitants. The maps reveal at a glance the most susceptible zones and the global vulnerability of the urban area in the different wind direction scenarios. From Fig. 6, it can be seen that the vulnerability index is not distributed homogeneously. On the contrary, the vulnerability tends to be maximal (red nodes) in a defined area of the map and its value gradually decreases in the nodes around it. Moreover, the position of the most vulnerable nodes varies strongly with small variations in the wind direction. As an example, notice the difference between the two scenarios related to $\Phi=0^{\circ}$ and $\Phi=45^{\circ}$ in Fig. 6 .

To better understand this behaviour, Fig. 7 a summaries the results of the sixteen simulated scenarios. Each scenario is represented by an arrow oriented with the wind direction, coloured according to the concentration ratio and positioned in the area of greatest vulnerability for that scenario. It is evident that for $\Phi$ equal to $0^{\circ}, 90^{\circ}, 180^{\circ}$ and $270^{\circ}$ (the cardinal directions) the most vulnerable nodes are located in the southern part of the network, while for $\Phi$ equal to $45^{\circ}, 135^{\circ}, 225^{\circ}$ and $315^{\circ}$ (the transversal directions) the most vulnerable areas are in the northern part of the network.

As the geometrical characteristics of the street canyons and the population density are rather homogeneously distributed in the street network, this different vulnerability pattern is related to the orientation of the streets with respect to the wind direction. In fact, the northern part of the street network is mainly oriented according to the cardinal directions (North-South and West-East oriented streets) and experiences the highest vulnerability when the wind blows according to one of the transversal directions. Conversely, the southern part of the street network is oriented mainly according to the transversal directions, and a greater vulnerability occurs when the wind is blowing in the cardinal directions. Thus, if the wind blows obliquely with respect to street orientations 

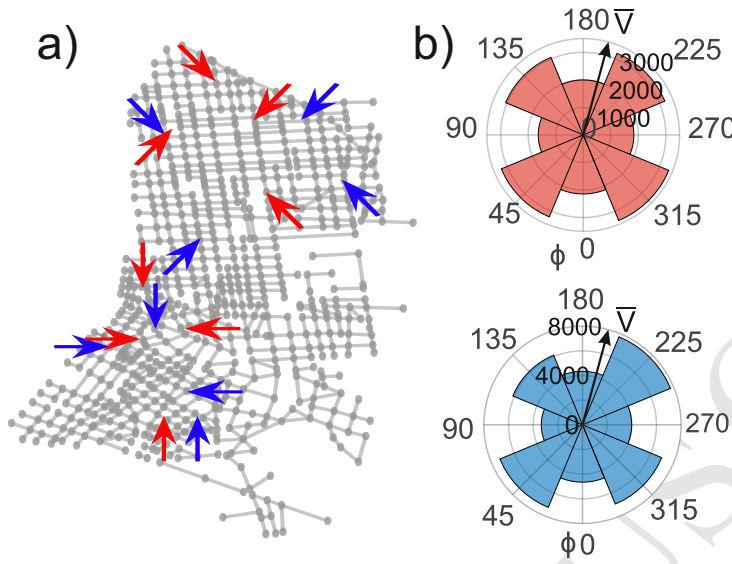

Figure 7: a) Most vulnerable areas in the street network for different wind directions and concentration ratios. Each scenario is represented by an arrow oriented according to the wind direction, coloured according to the concentration ratio (red arrows for $c_{0} / c_{t h}=10$ and blue arrows for $c_{0} / c_{t h}=100$ ) and positioned in the area of greatest vulnerability on the map. b) Polar histogram (red for $c_{0} / c_{t h}=10$ and blue for $c_{0} / c_{t h}=100$ ) of the average node vulnerability for the different wind directions

then vulnerability increases.

For each of the sixteen scenarios analysed, the average vulnerability over the entire network $(\bar{V})$ is calculated as

$$
\bar{V}=\frac{1}{N} \sum_{i}^{N} V_{i},
$$

where $V_{i}$ is the vulnerability index for the $i-t h$ node and $N$ is the number of nodes in the network. The two polar histograms of Fig. 7.b report these average vulnerability values for the two concentration ratios considered. Each sector in the polar histograms refers to a different wind direction. As expected, higher concentration ratios correspond to higher vulnerability values. Moreover, since most of the streets $(\sim 60 \%)$ are oriented in the cardinal directions, the average vulnerability is greater when the wind blows in the transversal directions.

Fig. 8 gives an overview of the long-term vulnerability of the urban fabric to toxic releases. The maps depict nodes vulnerability weighted by the annual 

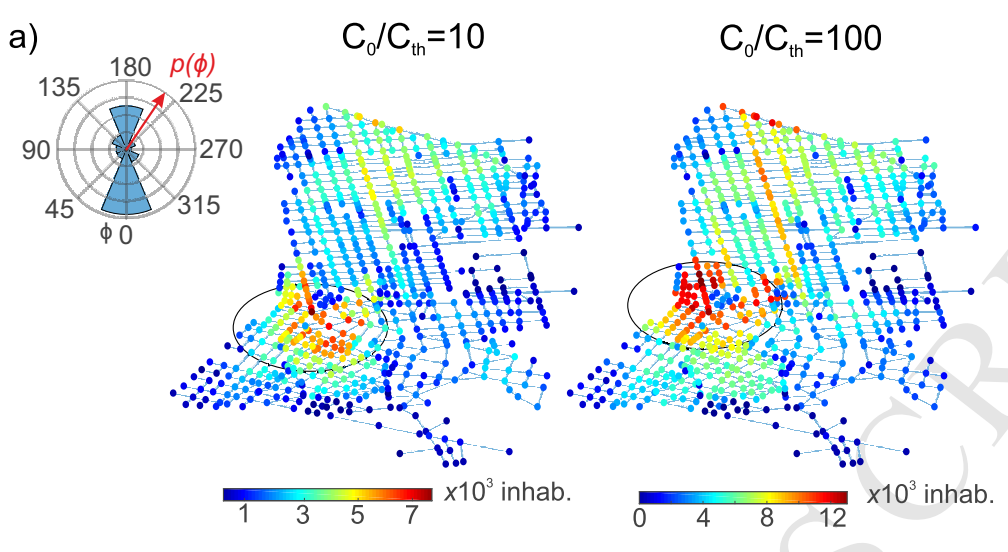

Figure 8: Maps of vulnerability weighted by the annual frequency of the wind directions. The polar histogram (inset $a$ ) shows the occurrence of wind directions in the city of Lyon in terms of annual relative frequency $p(\Phi)$.

frequency of the wind directions over the city of Lyon. As detailed in the inset of Fig. 8, the dominant wind direction in Lyon is North-South. As a consequence, the highest vulnerabilities are located in the circled area, corresponding to the most critical area when North-South oriented winds blow (see $\Phi=0^{\circ}$ and $\Phi=180^{\circ}$ scenarios in the supplementary material).

Results in Fig. 6] suggest that, for each scenario, a restricted area of the urban fabric is characterized by high levels of vulnerability, while most of the nodes have a low spreading potential. Thus, both spatial and frequency distribution of node vulnerabilities are not trivial. A frequency analysis of vulnerability values was performed to identify the statistical distribution of the data. For each scenario, node vulnerability values were classified in ten equal size intervals. Then, the relative frequency $(p)$ of each class was calculated. Fig. 9 presents the relative frequency of node vulnerability for the different scenarios in a loglog plot. For the sake of graphic clarity, vulnerability values were normalised to the maximum one $\left(V_{\max }\right)$. Generally, the data show a linear behaviour in the $\log -\log$ plot, thus exhibiting a power law trend. The power law confirms that vulnerability distribution is heterogeneous, with few nodes being much more critical than the others. This configuration suggests that, for each wind scenario, the entire neighbourhood could be protected with security interventions targeted 


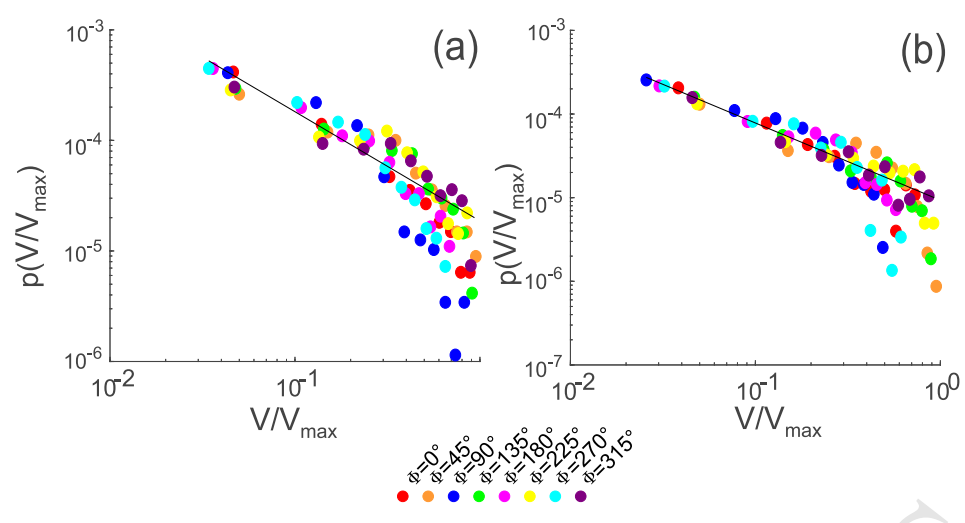

(b)

Figure 9: Relative frequency of vulnerability for different wind directions and for a concentration ratio $c_{0} / c_{t h}=10(\mathrm{a})$ and $c_{0} / c_{t h}=100(\mathrm{~b})$. The display is in logarithmic scale.

on small urban areas. From the opposite point of view, it demonstrates that a malicious release of gaseous substances can have a much more dramatic effect if it occurs in points characterized by maximum vulnerability.

Notice that many real-world networks (e.g., the Internet, World Wide Web, scientific citations) present a power law distribution of nodes degree (Boccaletti et al. 2006). The degree defines the importance of a node in the network in terms of its connectivity. In this case, a power law results from the nontrivial interaction of multiple factors that define the vulnerability index, i.e. the topological connectivity of the network, the geometry characteristics of the street canyons, the toxic spreading process and the population distribution in the city.

\section{Conclusions}

We have presented a complex network approach for the study of diffusion processes in the urban atmosphere. Within the urban canopy, the wind flows along the street canyons and the transport of pollutants is strongly influenced by the structure of the city, i.e. by the orientation of the streets and by their interconnections. Based on these considerations, we have modelled the interaction between the city and the external wind as a weighted complex network whose links and nodes represent the streets and the street intersections, respec- 
tively. The direction of the links and their weights describe the direction and the intensity of the wind along the streets and the geometrical properties of the buildings that surround the street canyons. Using a depth-first search analysis, we have implemented a spreading model on networks that simulates the propagation process from a point source. As an example, the developed method has been adopted to create vulnerability maps of the city of Lyon (France). These maps highlight the most vulnerable areas of the city, i.e. the areas from which the spread of a toxic substance, released at ground level, can harm more people. We found that the spatial and frequency distribution of urban vulnerability is heterogeneous and is strongly influenced by the alignment between the direction of the external wind and the orientation of the streets.

The model proved to be fast-to-run and functional, and therefore useful for the analysis of multiple scenarios that take into account various meteorological conditions, or different distributions of the residing population, and also temporary or permanent changes in the urban structure (e.g., the construction of a new building or the raising of a temporary wall to close a street during a public event). In particular, the computational time was estimated to be up to 3 orders of magnitude lower than that of the well-known street network model SIRANE. Thanks to these characteristics, this method is appropriate for the prediction and management of emergency scenarios, due to accidental or harmful releases of toxic substances in the urban atmosphere.

This work is in line with the efforts made in recent years (Hamlyn et al. 2007, Soulhac et al. 2011; Belcher et al., 2015; Goulart et al., 2018) to develop operational modelling tools for the prediction of pollutant transport in large urban areas. Moreover, the study demonstrate that the theory of complex networks can be adopted to this aim. The rate at which new techniques and applications are developed in the field of complex networks (in particular, in the field of epidemic spreading on networks) makes this new perspective particularly promising.

Future work is aimed at comparing different cities to understand how urban topology influences the dispersion and therefore the vulnerability of the city. In 
this way, it will be possible to understand which cities are the most structurally fragile. Further analyses should be conducted to consider variations in urban vulnerability due to changes in the presence of people in the different times of the day or during the different periods of the week. Finally, the results of this proof of concept study encourage the use of metrics and tools from complex network theory (e.g., centrality metrics) to investigate the role of the different key variables involved in urban dispersion, in order to better understand the fundamental mechanisms that drive this phenomenon.

\section{Appendix A. Longitudinal wind velocity along a street canyon}

According to Soulhac et al. (2008), the average velocity $u_{s t}$ along the longitudinal axis of a street canyon reads

$$
\begin{aligned}
& u_{s t}=u_{H} \cos \Phi \frac{\delta_{i}^{2}}{h w}\left[\frac{2 \sqrt{2}}{C}(1-\beta)\left(1-\frac{C^{2}}{3}+\frac{C^{4}}{45}\right)+\beta \frac{2 \alpha-3}{\alpha}\right. \\
& \left.+\left(\frac{w}{\delta_{i}}-2\right) \frac{\alpha-1}{\alpha}\right]
\end{aligned}
$$

$$
\text { with }=\left\{\begin{array}{l}
\alpha=\ln \left(\frac{\delta_{i}}{z_{0, \text { build }}}\right) \\
\beta=\exp \left[\frac{C}{\sqrt{2}}\left(1-\frac{h}{\delta_{i}}\right)\right] \\
u_{H}=u_{*} \sqrt{\frac{\pi}{\sqrt{2} k^{2} C}\left[Y_{0}(C)-\frac{J_{0}(C) Y_{1}(C)}{J_{1}(C)}\right]} \\
C \text { solution of } \frac{z_{0, \text { build }}}{\delta_{i}}=\frac{2}{C} \exp \left[\frac{\pi}{2} \frac{Y_{1}(C)}{J_{1}(C)}-\gamma\right] \\
\delta_{i}=\min \left(h, \frac{w}{2}\right)
\end{array}\right.
$$

where $\Phi$ is the external wind direction with respect to the street longitudinal axis, $h$ and $w$ are the height and the width of the street canyon, $z_{0, \text { build }}$ is the aerodynamic roughness of the canyon walls, $u_{*}$ is the friction velocity of the external atmospheric boundary layer flow, $J_{0}, J_{1}, Y_{0}$ and $Y_{1}$ are Bessel functions, $k$ is the von Kármán constant, and $\gamma$ is the Euler constant. The friction velocity $u_{*}$ is determined using the Monin-Obukhov similarity theory to model the flow 
in the external boundary layer.

\section{Appendix B. Solution for the one-dimensional transport equation}

Under the assumption that $c_{e x t}$ is negligible with respect to the concentration in the street canyon, the one-dimensional transport equation (1) becomes

$$
\frac{\partial c}{\partial t}+u_{s t} \frac{\partial c}{\partial x}+\frac{u_{d}}{h} c=0
$$

${ }_{67}$ By introducing the substitution

$$
g(x, t)=c(x, t) \exp \left(\frac{u_{d}}{h} t\right)
$$

B.1) yields

$$
\frac{\partial g}{\partial t}+u_{s t} \frac{\partial g}{\partial x}=0
$$

The general solution for $(\mathrm{B} .3)$ is found by introducing the new coordinates

$\tau=t, \quad \xi=x-u_{s t} t$ and using the chain rule:

$$
\begin{array}{r}
\frac{\partial g(x, t)}{\partial t}=\frac{\partial g(\xi, \tau)}{\partial \tau}-u_{s t} \frac{\partial g(\xi, \tau)}{\partial \xi} \\
\frac{\partial g(x, t)}{\partial x}=\frac{\partial g(\xi, \tau)}{\partial \xi}
\end{array}
$$

Equation B.3 becomes

$$
\frac{\partial g(\xi, \tau)}{\partial \tau}=0
$$

and, therefore,

$$
g(\xi, \tau)=F(\xi) \quad \rightarrow \quad g(x, t)=F\left(x-u_{s t} t\right),
$$


where $F$ is a derivable function. The solution of $(\mathrm{B} .1)$ is obtained returning to the original function,

$$
c(x, t)=g(x, t) \exp \left(-\frac{u_{d}}{h} t\right)=F\left(x-u_{s t} t\right) \exp \left(-\frac{u_{d}}{h} t\right) .
$$

Function $F$ is found using the initial or boundary conditions of the problem.

For a continuous release $c_{0}$ in the source node starting from $t \geq 0$, the boundary condition is defined as

$$
c(0, t)=c_{0} \Theta(t)
$$

where $\Theta$ is the Heaviside function. For $x=0,(B .8)$ and $(\overline{B .9})$ yield

$$
c(0, t)=F\left(-u_{s t} t\right) \exp \left(-\frac{u_{d}}{h} t\right)=c_{0} \Theta(t) .
$$

Function $F$ is obtained from the previous one and the solution for the continuous release is thus

$$
c(x, t)=F\left(x-u_{s t} t\right) \exp \left(-\frac{u_{d}}{h} t\right)=c_{0} \exp \left(-\frac{u_{d}}{u_{s t}} \frac{x}{h}\right) \Theta\left(t-\frac{x}{u_{s t}}\right) .
$$

For a quick release in the source node, the initial condition is set as a rectangular pulse with width $a$ and height $c_{0}$

$$
c(x, 0)=c_{0}[\Theta(x)-\Theta(x-a)] .
$$

When $a$ tends to zero, the release is almost instantaneous. Following the same reasoning as in $\mathrm{B} .10$ and $\mathrm{B} .11$, but considering this time $t=0$, we obtain the solution for a quick release:

$$
c(x, t)=c_{0} \exp \left(-\frac{u_{d}}{h} t\right)\left[\Theta\left(x-u_{s t} t\right)-\Theta\left(x-u_{s t} t-a\right)\right] .
$$




\section{Appendix C. Adjacency matrix}

Consider the network in Fig11;, extracted as a subgraph from the network of streets in Fig 1 1 . The adjacency matrix of this simple four-links graph reads

$$
\mathbf{A}=\begin{gathered}
s \\
s \\
g \\
i \\
i \\
k \\
k
\end{gathered}\left(\begin{array}{llllll}
0 & 0 & 1 & 0 & 0 \\
0 & 0 & 1 & 0 & 0 \\
0 & 0 & 0 & 1 & 1 \\
0 & 0 & 0 & 0 & 0 \\
0 & 0 & 0 & 0 & 0
\end{array}\right) .
$$

Note that the matrix is asymmetric since the network is directed (i.e. the links have a specific direction).

\section{References}

Ahern, J., 2011. From fail-safe to safe-to-fail: Sustainability and resilience in the new urban world. Landscape and Urban Planning 100 (4), 341-343.

Arnfield, A. J., 2003. Two decades of urban climate research: A review of turbulence, exchanges of energy and water, and the urban heat island. International Journal of Climatology 23 (1), 1-26.

Barthélemy, M., 2011. Spatial networks. Physics Reports 499 (1-3), 1-101.

Belcher, S., Coceal, O., Goulart, E., Rudd, A., Robins, A., 2015. Processes controlling atmospheric dispersion through city centres. Journal of Fluid Mechanics $763,51-81$.

Berke, P. R., Song, Y., Stevens, M., 2009. Integrating hazard mitigation into new urban and conventional developments. Journal of Planning Education and Research 28 (4), 441-455. 
Blocken, B., 2015. Computational Fluid Dynamics for urban physics: Importance, scales, possibilities, limitations and ten tips and tricks towards accurate and reliable simulations. Building and Environment 91, 219-245.

Boccaletti, S., Latora, V., Moreno, Y., Chavez, M., Hwang, D.-U., 2006. Complex networks: Structure and dynamics. Physics Reports 424 (4-5), 175-308.

Borgatti, S. P., Mehra, A., Brass, D. J., Labianca, G., 2009. Network analysis in the social sciences. Science 323 (5916), 892-895.

Brunekreef, B., Holgate, S. T., 2002. Air pollution and health. The Lancet 360 (9341), 1233-1242.

Carruthers, D., Edmunds, H., Lester, A., McHugh, C., Singles, R., 2000. Use and validation of ADMS-Urban in contrasting urban and industrial locations. International Journal of Environment and Pollution 14 (1-6), 364-374.

Carvalho, R., Buzna, L., Bono, F., Gutiérrez, E., Just, W., Arrowsmith, D., 2009. Robustness of trans-European gas networks. Physical Review E 80 (1), 016106.

Comin, C. H., da Fontoura Costa, L., 2011. Identifying the starting point of a spreading process in complex networks. Physical Review E 84 (5), 056105.

Di Sabatino, S., Buccolieri, R., Salizzoni, P., 2013. Recent advancements in numerical modelling of flow and dispersion in urban areas: A short review. International Journal of Environment and Pollution 752 (3-4), 172-191.

Gelbrecht, M., Boers, N., Kurths, J., 2017. A complex network representation of wind flows. Chaos: An Interdisciplinary Journal of Nonlinear Science 27 (3), 035808 .

Giustolisi, O., Ridolfi, L., 2014. A novel infrastructure modularity index for the segmentation of water distribution networks. Water Resources Research 50 (10), 7648-7661. 
Goulart, E. V., Coceal, O., Belcher, S. E., 2018. Dispersion of a passive scalar within and above an urban street network. Boundary-Layer Meteorology $166(3), 351-366$.

Hamlyn, D., Hilderman, T., Britter, R., 2007. A simple network approach to modelling dispersion among large groups of obstacles. Atmospheric Environment 41 (28), 5848-5862.

Heinrich, J., Wichmann, H.-E., 2004. Traffic related pollutants in Europe and their effect on allergic disease. Current Opinion in Allergy and Clinical Immunology 4 (5), 341-348.

Hunter, L. J., Watson, I., Johnson, G., 1990. Modelling air flow regimes in urban canyons. Energy and Buildings 15 (3-4), 315-324.

Kim, Y., Wu, Y., Seigneur, C., Roustan, Y., 2018. Multi-scale modeling of urban air pollution: development and application of a street-in-grid model (v1.0) by coupling munich (v1. 0) and polair3d (v1. 8.1). Geoscientific Model Development 11 (2), 611-629.

Kozen, D. C., 1992. Depth-first and breadth-first search. In: The Design and Analysis of Algorithms. Springer, pp. 19-24.

Mayer, H., 1999. Air pollution in cities. Atmospheric Environment 33 (24-25), 4029-4037.

McLeish, C., 2017. Recasting the threat of chemical terrorism in the EU: The issue of returnees from the syrian conflict. European Journal of Risk Regulation 8 (4), 643-657.

Namdeo, A., Colls, J., 1996. Development and evaluation of SBLINE, a suite of models for the prediction of pollution concentrations from vehicles in urban areas. Science of the Total Environment 189, 311-320.

Newman, M., 2010. Networks: An introduction. Oxford university press. 
Newman, M. E., 2002. Spread of epidemic disease on networks. Physical Review E 66 (1), 016128.

Oke, T. R., 1982. The energetic basis of the urban heat island. Quarterly Journal of the Royal Meteorological Society 108 (455), 1-24.

Porta, S., Crucitti, P., Latora, V., 2006. The network analysis of urban streets: A dual approach. Physica A: Statistical Mechanics and its Applications $369(2), 853-866$.

Robins, A., Savory, E., Scaperdas, A., Grigoriadis, D., 2002. Spatial variability and source-receptor relations at a street intersection. Water, Air and Soil Pollution: Focus 2 (5-6), 381-393.

Roth, M., 2000. Review of atmospheric turbulence over cities. Quarterly Journal of the Royal Meteorological Society 126 (564), 941-990.

Salizzoni, P., Soulhac, L., Mejean, P., 2009. Street canyon ventilation and atmospheric turbulence. Atmospheric Environment 43 (32), 5056-5067.

Scaperdas, A.-S., 2000. Modelling air flow and pollutant dispersion at urban canyon intersections.

Ser-Giacomi, E., Rossi, V., López, C., Hernandez-Garcia, E., 2015. Flow networks: A characterization of geophysical fluid transport. Chaos: An Interdisciplinary Journal of Nonlinear Science 25 (3), 036404.

Soulhac, L., 2000. Modélisation de la dispersion atmosphérique à lintérieur de la canopée urbaine. Ph.D. thesis, Ecole Centrale de Lyon.

Soulhac, L., Garbero, V., Salizzoni, P., Mejean, P., Perkins, R., 2009. Flow and dispersion in street intersections. Atmospheric Environment 43 (18), 29812996.

Soulhac, L., Perkins, R. J., Salizzoni, P., 2008. Flow in a street canyon for any external wind direction. Boundary-Layer Meteorology 126 (3), 365-388. 
Soulhac, L., Salizzoni, P., Cierco, F.-X., Perkins, R., 2011. The model SIRANE for atmospheric urban pollutant dispersion; part I, presentation of the model. Atmospheric Environment 45 (39), 7379-7395.

Soulhac, L., Salizzoni, P., Mejean, P., Perkins, R., 2013. Parametric laws to model urban pollutant dispersion with a street network approach. Atmospheric Environment 67, 229-241.

Tominaga, Y., Stathopoulos, T., 2012. CFD modeling of pollution dispersion in building array: Evaluation of turbulent scalar flux modeling in RANS model using LES results. Journal of Wind Engineering and Industrial Aerodynamics 104, 484-491.

Tucker, J. B., 2000. Toxic terror: Assessing terrorist use of chemical and biological weapons. MIT Press.

Tzella, A., Vanneste, J., 2016. Dispersion in rectangular networks: effective diffusivity and large-deviation rate function. Physical review letters 117 (11), 114501.

Valiente, G., 2013. Algorithms on trees and graphs. Springer Science \& Business Media.

Yazdani, A., Jeffrey, P., 2011. Complex network analysis of water distribution systems. Chaos: An Interdisciplinary Journal of Nonlinear Science 21 (1), 016111. 
- A complex network approach to model dispersion in the urban atmosphere is presented

- Dispersion from a source is modelled as a spreading process on a complex network

- The polluted area and the number of people affected by the release are assessed

- Maps of urban vulnerability to the release of toxic gases are rapidly constructed

- The street topology of the city is a key driver in the dispersion process 